\title{
The value of virtual bronchoscopic navigation and radial endobronchial ultrasound-guided transbronchial lung cryobiopsies for pulmonary lymphangitic carcinomatosis
}

\author{
Lingling Pang", Tingshu Jiang", Xueping Liu, Zhan Li, Shenchun Zou, Pengfei Yu \\ Department of Pulmonary and Critical Care Medicine, Yantai Yuhuangding Hospital, Yantai, China \\ Contributions: (I) Conception and design: L Pang, P Yu; (II) Administrative support: T Jiang, S Zou, P Yu; (III) Provision of study materials or \\ patients: T Jiang, Z Li; (IV) Collection and assembly of data: L Pang, X Liu; (V) Data analysis and interpretation: L Pang; (VI) Manuscript writing: \\ All authors; (VII) Final approval of manuscript: All authors. \\ \#These authors contributed equally to this work. \\ Correspondence to: Pengfei Yu. Department of Pulmonary and Critical Care Medicine, Yantai Yuhuangding Hospital, Yantai 264000, China. \\ Email: yhdlish@163.com.
}

Background: Pulmonary lymphangitic carcinomatosis (PLC) is characterized by malignant infiltration into lung lymphatic channels from a primary site and is often observed in advanced malignant tumors. This study aimed to evaluate the diagnostic yield of transbronchial lung cryobiopsy in PLC guided by radial endobronchial ultrasound and virtual bronchoscopic navigation (VBN).

Methods: This prospective study enrolled 40 patients with clinical and radiologic features indicating PLC. The radial endobronchial ultrasound probe was initially advanced to the region of interest of the desired lobe near the pleura with guidance by VBN. Transbronchial lung biopsy and transbronchial lung cryobiopsy were both performed in the same ROI of all patients with the obtained samples being sent to the pathology laboratory for diagnostic analysis. Procedural complications were recorded.

Results: The average number of transbronchial lung biopsy and transbronchial lung cryobiopsy specimens were 4 ( 3 to 6 ) and 2 ( 1 to 3 ), respectively ( $\mathrm{t}=10.43, \mathrm{P}<0.01$ ), with the corresponding mean diameters per biopsy being 3.7 and $8.7 \mathrm{~mm}(\mathrm{t}=12.37, \mathrm{P}<0.01)$. The diagnostic yields of transbronchial lung biopsy and transbronchial lung cryobiopsy were 70\% (28/40) and $92.5 \%$ (37/40), respectively. The final positive predictive values of transbronchial lung cryobiopsy and transbronchial lung biopsy for PLC were $94.4 \%$ $(34 / 36)$ and $77.8 \%(28 / 36)$, respectively $\left(\chi^{2}=23.94, \mathrm{P}<0.01\right)$. Further, 52.2\% (12/23) and 81.5\% $(22 / 27)$ of the patients in the transbronchial lung biopsy and transbronchial lung cryobiopsy groups, respectively, were diagnosed with non-small lung cancer after further molecular analysis $\left(\chi^{2}=19.56, \mathrm{P}<0.01\right)$. Only $2(5 \%)$ cases presented postoperative pneumothorax. Moreover, $0(0 \%), 3(7.5 \%)$, and $17(42.5 \%)$ patients presented severe, moderate, and mild bleeding, respectively. There were no other adverse events or deaths.

Conclusions: Transbronchial lung cryobiopsy with the guidance of radial endobronchial ultrasound and VBN without fluoroscopy has a good diagnostic yield for PLC; moreover, it allows one to obtain adequate and intact tissue samples for further molecular analysis.

Keywords: Pulmonary lymphangitic carcinomatosis (PLC); transbronchial lung cryobiopsy; transbronchial lung biopsy; radial endobronchial ultrasound; virtual bronchoscopic navigation (VBN)

Submitted Nov 16, 2020. Accepted for publication Dec 17, 2020.

doi: $10.21037 /$ jtd-2020-abpd-002

View this article at: http://dx.doi.org/10.21037/jtd-2020-abpd-002

(C) Journal of Thoracic Disease. All rights reserved. 


\section{Introduction}

Pulmonary lymphangitic carcinomatosis (PLC) is characterized by malignant infiltration into lung lymphatic channels from a primary site and generally originates from adenocarcinoma of the lung, breast, stomach, prostate, and pancreas (1). Specifically, it is an unusual presentation of diffuse infiltrative lung disease with nonspecific symptoms, which often results in a delayed diagnosis. High resolution computed tomography (HRCT) is a vital method for PLC diagnosis with the characteristic radiologic features including thickened peribronchovascular interstitium and interlobular septa, subpleural edema, pulmonary nodules with accompanying bronchovascular bundles, hilar and mediastinal nodal enlargement, pleural effusion, and slight destruction of the overall lung architecture (2-4). Clinical PLC diagnosis is often difficult given the lack of specific respiratory complaints and radiologic features. Half of the patients with PLC die within 3 months after respiratory symptom onset (5); moreover, poor prognosis requires early histopathologic diagnosis and treatment.

Despite the well-known high diagnostic value of thoracic surgery lung biopsy $(6,7)$, patients may require a few postoperative days of hospitalization and develop numerous complications associated with mortality risk (6-8). TBLB using endoscopic forceps is the most common diagnostic approach. However, the tissue sample size obtained by standard TBLB usually limits the diagnostic yield $(9,10)$. The TBLB diagnostic yield has been reported to be $<70 \%$ and $29 \%$ for peripheral pulmonary lesions (PPL) (11) and interstitial lung disease (12), respectively.

Since the 1970s, cryoprobes have been employed in bronchoscopy. When the probe tip is cooled by releasing the compressed carbon dioxide $\left(\mathrm{CO}_{2}\right)$ or nitric oxide $(\mathrm{NO})$, the tissue adjacent to the probe is attached to the frozen probe and isolated. Over the last decade, TBLC has been performed to obtain sufficient specimens for the diagnosis of interstitial lung diseases (ILD), which could explain the higher diagnostic yield compared with that of TBLB $(10,12-18)$. However, there have been few studies on the role of TBLC on PLC.

Guidance techniques, including fluoroscopy, virtual bronchoscopic navigation (VBN), electromagnetic navigation bronchoscopy (ENB), and radial endobronchial ultrasound (R-EBUS), are applied to TBLC to enhance the diagnostic yield of PLC and avoid complications. R-EBUS is a minimally invasive bronchoscopic technique that is routinely used for PPL diagnosis (19). It generates a $360^{\circ}$ ultrasound field of view, which allows bronchoscopists to avoid blood vessels and to target the lung biopsy site. In our clinical practice, the "blizzard" pattern is manifested in PLC due to increased echogenicity resulting from interstitial tumor cell infiltration. R-EBUS is often combined with other guidance techniques to improve target localization. VBN is a navigation technique that employs CT images to reconstruct the virtual bronchial tree and provide a route to the target. VBN images are created based on imaging data obtained before bronchoscopy examination. The combination of VBN with R-EBUS has a higher diagnostic yield for PPL (20).

There have been few studies on PLC biopsy. This study aimed to assess the diagnostic yield of TBLC for PLC, as well as to evaluate the procedure-related complications.

We present the following article in accordance with the STARD reporting checklist (available at http://dx.doi. org/10.21037/jtd-2020-abpd-002).

\section{Methods}

\section{Patients}

This prospective study was conducted in the department of pulmonary and critical care, Yantai Yuhuangding Hospital from January 2017 to November 2018. The study was conducted in accordance with the Declaration of Helsinki (as revised in 2013). The study protocol was reviewed and approved by the Ethics Committee of Qingdao Medical College Affiliated to Yantai Yuhuangding Hospital (No. 2014-111), which waived the requirement for informed consent due to the retrospective nature of the study. All the patients provided written informed consent before treatment. This open-label study enrolled consecutive patients with pulmonary infiltrates on HRCT and suspected of PLC. Typical imaging features of PLC on HCRT include perihilar axial interstitium, thickened interlobular septa, intrapulmonary nodules, subpleural interstitium, and no structural lung lobule deformations. Moreover, we enrolled patients with additional imaging features of hilar and mediastinal nodal enlargement, as well as pleural effusion. Other inclusion criteria were: (I) age $>18$ years and $<80$ years; (II) platelet count $>80,000 / \mathrm{mm}^{3}$. The main exclusion criteria were: (I) standard contraindications to invasive procedures, including heart failure and unstable cardiovascular disease; (II) forced vital capacity $<50 \%$ predicted and diffusion capacity of carbon monoxide $<35 \%$ predicted; (III) abnormal coagulation test results; 

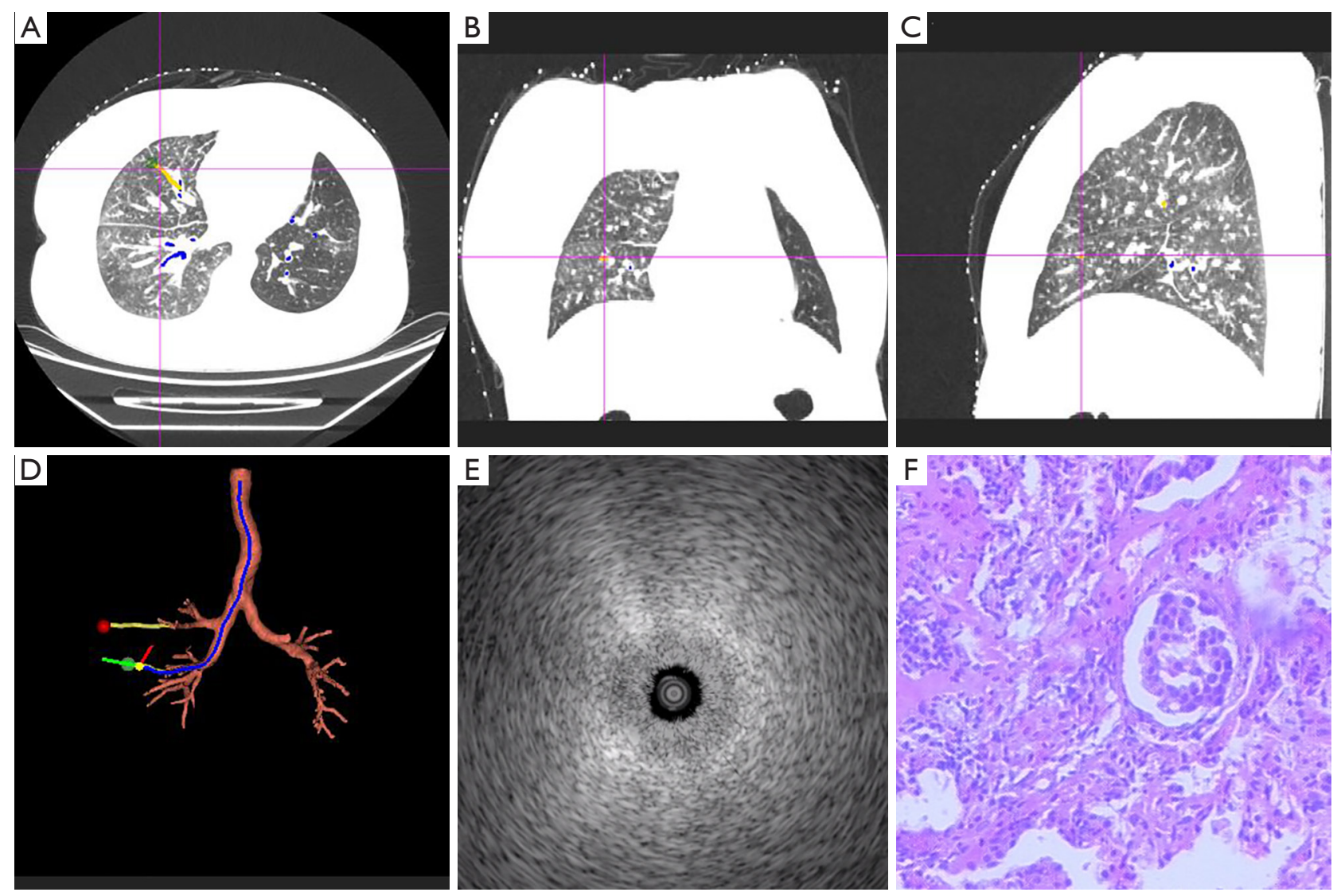

Figure 1 Images of a 54-year-old woman with PLC. HRCT of the axial position, coronal position, and sagittal positions (A,B,C). A 3D-reconstruction of the tracheobronchial tree by VBN (D). R-EBUS image showing a blizzard pattern (E). Cryobiopsy confirmed lung adenocarcinoma (F; HE, $\times 100)$.

(VI) having taken oral anticoagulants within 1 week; (V) contraindications to anesthesia; and (VII) pregnant women.

\section{Procedure and Equipment}

HRCT images were imported into the VBN system (DirectPath $^{\mathrm{TM}}$, Olympus, Tokyo, Japan); subsequently, one to three targets were selected in each patient at a distance of about $1 \mathrm{~cm}$ from the chest wall. The lower lung zones and lateral airways were prioritized. After target localization, the virtual airway pathway and distance for approaching the target was calculated using VBN (21). The VBN system was positioned beside the bronchoscopic screen.

TBLC was conducted in patients intubated with a rigid tube (size 8.5\# to 14\#; Karl Storz, Tuttlingen, Germany). An interventional pulmonologist performed flexible bronchoscopy with the patient under general anesthesia (22).

\section{$R$-EBUS guided TBLC procedure}

A bronchoscope (BF-P290; Olympus, Tokyo, Japan) was introduced through intubation and navigated to the target with guidance by the VBN system. Upon reaching the most distal airway, an ultrasound probe (20 MHz, UM-S20-17S; Olympus, Tokyo, Japan) was inserted through the working channel. This procedure did not involve fluoroscopy. The probe length and angle were based on previously calculated VBN data. The absence of major vessels and the presence of blizzard patterns were indicative of the safe area for biopsy (Figures 1,2). In case the desired pattern was not observed or was too close to the pleura, a biopsy sample was not obtained and another target area was explored. Once the optimal location was confirmed, the EBUS probe was removed followed by the introduction of transbronchial forceps into the area. Five biopsies were suggested during 

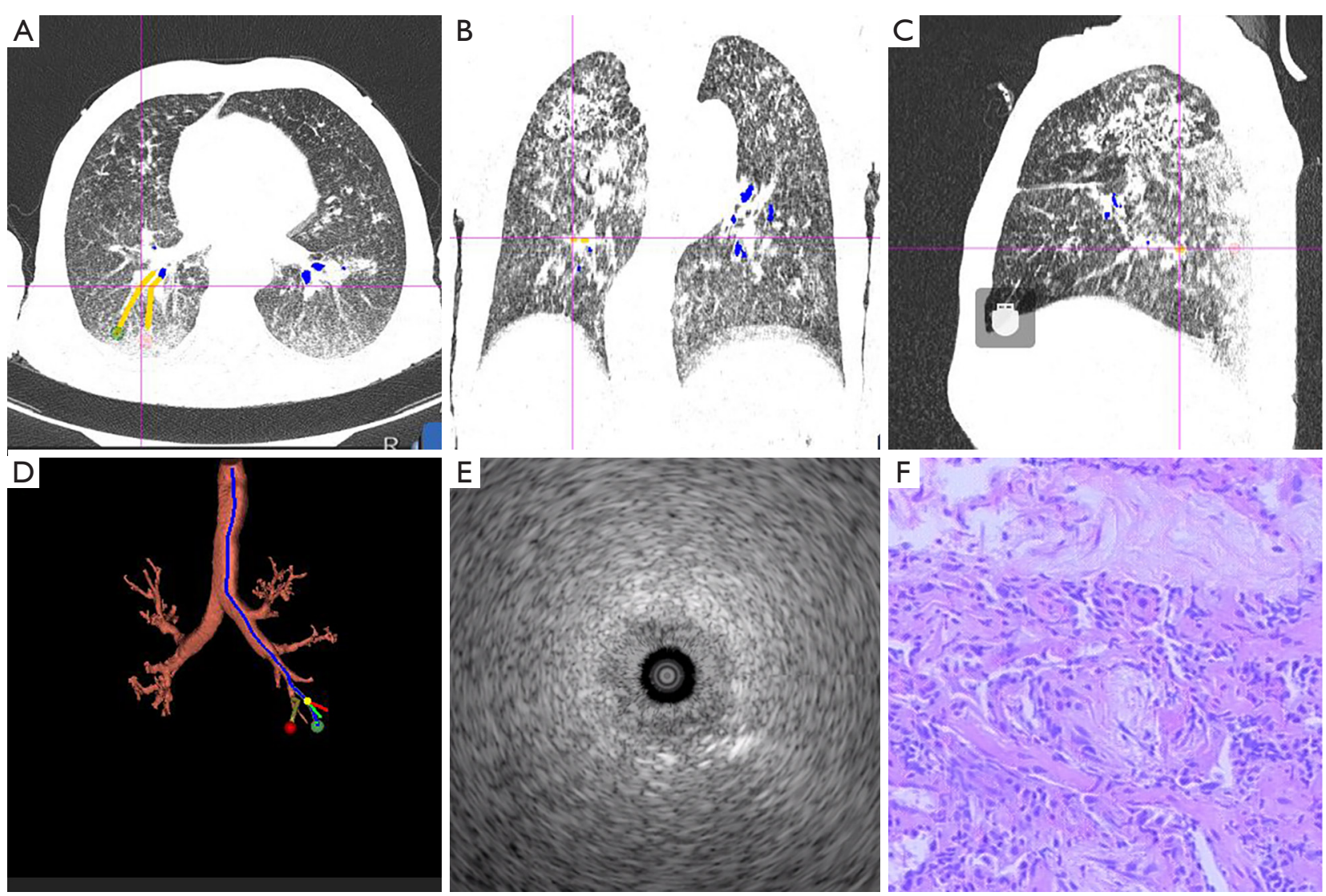

Figure 2 Images of a 70-year-old man suspected with PLC. HRCT of the axial, coronal, and sagittal positions (A,B,C). A 3D-reconstruction of tracheobronchial tree by VBN (D). R-EBUS image showing a blizzard pattern (E). Cryobiopsy confirmed interstitial pulmonary tuberculosis (F; HE, $\times 100)$.

the procedure. The cryoprobe $(1.8 \mathrm{~mm}$ diameter; Kooland, Beijing, China) was inserted into the same target segmental bronchi as the previous TBLB. The cryoprobe was activated for 5-6 s; subsequently, it was quickly removed together with the bronchoscope. The tissue was thawed from the cryoprobe in normal saline and transferred to formalin. Two biopsies were suggested. Finally, the bronchoscope was re-advanced into the airway for bleeding assessment and management. A balloon catheter had been previously prepared for possible major bleeding. TBLB and TBLC specimens were sent to the department of pathology respectively. A pathologist reviewed all specimens and recorded whether the final pathologic diagnostic results were confirmed using TBLB and/or TBLC specimens. Moreover, whether the specimens were suitable for further genetic analysis was recorded.

\section{Complications}

Cryobiopsy-related bleeding was categorized into mild (requiring bronchoscopic suctioning), moderate (requiring intrabronchial administration of epinephrine or ice-cold saline), and severe (requiring procedure termination, blood transfusion, or prolonged intubation requiring intensive care unit monitoring) (23). All the patients underwent chest $\mathrm{X}$-ray within 2 postoperative hours to evaluate procedurerelated pneumothorax, as well as other complications.

\section{Statistical analysis}

Baseline characteristics of the patients were analyzed using descriptive statistics. Normally and non-normally distributed continuous variables were expressed as medians 
with standard deviations and median [interquartile range (IQR)], respectively. Categorical data were expressed as percentages and frequencies. The diagnostic yield was calculated using the pathologic diagnostic results. Betweengroup comparisons of categorical variables were performed using a $t$-test or Chi-squared test. Statistical significance was set at two-sided $\mathrm{P}<0.05$.

Table 1 Patient characteristics of study population

\begin{tabular}{lc}
\hline Subjects & Data \\
\hline Age (years) & $62.5(41.7-78.3)$ \\
Sex (male/female) & $26 / 14$ \\
Smoking history (\%) & $67.5 \%(27 / 40)$ \\
Upper lobe (\%) & $7.5 \%(3 / 40)$ \\
Middle lobe (\%) & $20 \%(8 / 40)$ \\
Lower lobe (\%) & $72.5 \%(29 / 40)$ \\
Inner third (\%) & $2.5 \%(1 / 40)$ \\
Middle third (\%) & $12.5 \%(5 / 40)$ \\
Outer third (\%) & $85 \%(34 / 40)$ \\
Past malignancy (\%) & $7.5 \%(3 / 40)$ \\
FEV1 (L) & $1.81 \pm 0.07$ \\
FVC predicted (\%) & $0.65 \pm 0.01$ \\
\hline
\end{tabular}

Data were presented as $\%(n / N)$, mean $\pm S D$, or median $(I Q R)$. FEV1, forced expiratory volume in 1 second; FVC, forced vital capacity.

\section{Results}

From January 2017 to November 2018, we enrolled 40 patients. The overall median (IQR) age of patients was 62.5 (41.7-78.3) years; further, $65.0 \%$ of the patients were male. Most lesions were located in the outer third of the hemithorax in the lower lobe. Table 1 presents the baseline clinical and lesion characteristics.

The average number of TBLB and TBLC specimens were 4 ( 3 to 6$)$ and 2 ( 1 to 3$)$, respectively, ( $\mathrm{t}=10.43$, $\mathrm{P}<0.01)$, with the corresponding mean diameters per biopsy being 3.7 and $8.7 \mathrm{~mm}(\mathrm{t}=12.37, \mathrm{P}<0.01)$ (Figure 3$)$. In the VBN and R-EBUS guided TBLB group, malignancies were identified in $28(70 \%)$ patients; among them, 20, 3, 3 , and 2 patients were diagnosed with adenocarcinoma, squamous cell lung cancer, small cell lung cancer, and other malignancies, respectively. These diagnoses were reached by both TBLB and TBLC. An additional 9 diagnoses were established through TBLC, which increased the overall diagnostic yield to $92.5 \%$ (37/40). Among the 9 patients, $4,2,2$, and 1 were diagnosed with adenocarcinoma, small cell lung cancer, tuberculosis, and organizing pneumonia, respectively. Both TBLB and TBLC failed to achieve a diagnostic yield in 3 patients. Among them, a definitive diagnosis was established using video-assisted thoracoscopic surgery in 2 patients while a final diagnosis could not be reached in 1 patient due to progressive worse respiratory symptoms and arterial oxygen pressure. The final positive predictive value of TBLC and TBLB for malignancies were $94.4 \%(34 / 36)$ and $77.8 \%(28 / 36)$, respectively $\left(\chi^{2}=23.94\right.$,
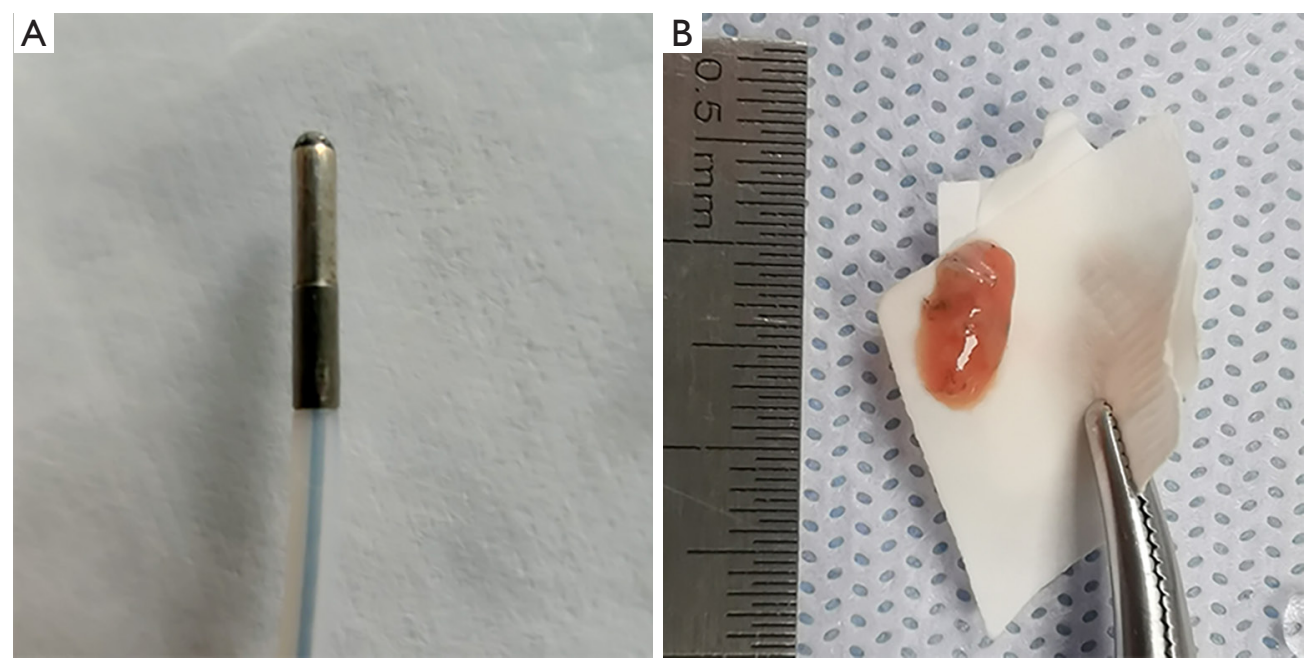

Figure 3 Image of a cryoprobe (A) and lung tissue specimen obtained by TBLC (B). 
Table 2 The procedure characteristics and diagnostic yield of TBLB and TBLC for PLC

\begin{tabular}{lccc}
\hline Variables & TBLB & TBLC & P value \\
\hline Biopsy number & 2 (1 to 3) & 4 (3 to 6) & 0.000 \\
Biopsy size (mm) & $3.7 \pm 0.04$ & $8.7 \pm 0.07$ & 0.015 \\
Adenocarcinoma & 20 & 24 & - \\
Squamous cell lung cancer & 3 & 3 & - \\
Small cell lung cancer & 3 & 5 & - \\
Other malignancies & 2 & 2 & - \\
Diagnostic yield & $77.8 \%$ & $94.4 \%$ & 0.003 \\
\hline
\end{tabular}

Data were presented as mean $\pm S D$, or median (IQR). Categorical variables of biopsy number and size between two groups were compared using $t$-test. Categorical variables of diagnostic yield were compared using Chi-squared test. $\mathrm{P}<0.05$ was considered statistically significant.

$\mathrm{P}<0.01)$. TBLB and TBLC specimens were sufficient for further genetic analysis in 52.2\% (12/23) and 81.5\% (22/27) of the patients, respectively, diagnosed with non-small lung cancer $\left(\chi^{2}=19.56, \mathrm{P}<0.01\right)$.

Only $2(5 \%)$ patients developed postoperative pneumothorax, with one patient requiring pleural drainage. There was no occurrence of severe life-threatening bleeding events; moreover, moderate, and mild bleeding occurred in $3(7.5 \%)$ and $17(42.5 \%)$ patients, respectively. There were no other adverse events or death. Table 2 presents the procedure characteristics and diagnostic yield of TBLB and TBLC for PLC.

\section{Discussion}

PLC is an unusual presentation of diffuse infiltrative lung disease with nonspecific symptoms, which often results in delayed diagnosis. Although HRCT and PETCT are crucially involved in identifying PLC $(3,4)$, there is a need to obtain pathology diagnosis to allow prompt treatment. Transbronchial biopsy is the most common and least invasive approach. The reported diagnostic yield for ILD ranges from $75 \%$ to $93 \%$ (24-26). In the present study, our diagnostic yield and adequate specimen rate for genetic analysis were $92.5 \%$ and $81.5 \%$, respectively, which was prior to those previously reported. It was thought that disorders distributed along the lymphatic routes, including OP and sarcoidosis, were easily identifiable $(25,26)$, and there might be the same result in PLC. The optimal cryobiopsy number remains unclear, with 2-5 biopsies being suggested (15). In this study, 2-3 biopsies were sufficient for pathological and molecular evaluation. The mean diameter of the samples we obtained was consistent with that previously reported $(24,27,28)$. The present study suggested that TBLC was quite qualified for diagnosing suspected PLC with 2-3 biopsies.

The main complications of TBLC include pneumothorax and massive hemoptysis. It is thought that the pneumothorax risk increases upon cryoprobe insertion into the subpleural area (21). For safety purposes, there is a need to determine if the freezing tip of the flexible cryoprobe is sufficiently distant from the chest wall during TBLC. In this study, we manually used the VBN system for target localization and to avoid the subpleural area based on lesion location. Subsequently, the VBN system calculated the potential pathway, and the bronchoscope could be guided into the third to eighth generation bronchi. The VBN system has been used to enhance the diagnostic yield of transbronchial biopsy for PPL (29,30). Currently, there has been no study evaluating the diagnostic yield of VBN for PLC; however, VBN did facilitate the selection of the correct bronchus to reach the target and shorten the procedure time. Further, the low pneumothorax incidence rate $(5 \%)$ in our study could be attributed to the use of VBN. A review literature reported that the pneumothorax incidence of TBLC was approximately $10 \%$ without VBN (31).

Since VBN is not a real-time imaging technique, we combined it with R-EBUS to choose the most proper biopsy site. Given that vessels are usually running along the bronchus in the peripheral lung, the risk of severe bleeding increases when the cryoprobe is too close to the pulmonary vessels, which can be avoided using an ultrasound probe. R-EBUS works with a guide sheath (GS), which serves as a path for sampling devices and facilitates post-biopsy bleeding control; consequently, the rate of bleeding complications is lower (32). We observed a high bleeding incidence mainly due to the absence of a GS during cryobiopsies since the cryoprobe could not pass through the GS. However, the use of R-EBUS led to no case of severe bleeding. TBLC was performed immediately after TBLB without chest $\mathrm{X}$-ray examination, and either abovementioned procedure could have resulted in complications, including pneumothorax. Nonetheless, the complication rate was not higher than that in literature, which is suggestive of the safety of TBLC.

In our study, the average age of the patients was 
62.5 years (range, 41.7-78.3 years), which suggests that TBLC is acceptable for elderly patients. For elderly patients with suspected PLC and progressive dyspnea, a diagnosis is difficult to establish since they cannot tolerate surgical lung biopsy or CT-guided transthoracic needle aspiration. Therefore, TBLC may be a promising diagnostic choice, and guidance with VBN and R-EBUS may improve the diagnostic yield and decrease the procedure-related complications.

There are several limitations in this study. First, since PLC is a relatively rare manifestation of metastatic cancer, our sample size was relatively small and this was a singlecenter study. Second, the use of TBLB and TBLC in the same patient might have increased the procedure-related risk; additionally, it was difficult to identify the operation responsible for the risk. There is a need for a randomized controlled trial to evaluate the diagnostic rate and risk of TBLB and TBLC.

\section{Conclusions}

In summary, our findings indicate that TBLC could increase the diagnostic yield of PLC with safety assurance through guidance using VBN and R-EBUS. Randomized controlled trials should evaluate the diagnostic rate and risk of TBLB and TBLC in the future.

\section{Acknowledgments}

Funding: The present study was supported by a grant from the Science and Technology Planning Project of Yantai (grant no. 2020YD004 to PY and grant no. 2020YD082 to LP).

\section{Footnote}

Provenance and Peer Review: This article was commissioned by the Guest Editor (Jiayuan Sun) for the series "Advance in Bronchoscopy for Peripheral Pulmonary Diseases" published in Fournal of Thoracic Disease. The article has undergone external peer review.

Reporting Checklist: The authors have completed the STARD reporting checklist. Available at http://dx.doi.org/10.21037/ jtd-2020-abpd-002

Data Sharing Statement: Available at http://dx.doi. org/10.21037/jtd-2020-abpd-002
Conflicts of Interest: All authors have completed the ICMJE uniform disclosure form (available at http://dx.doi. org/10.21037/jtd-2020-abpd-002). The series "Advance in Bronchoscopy for Peripheral Pulmonary Diseases" was commissioned by the editorial office without any funding or sponsorship. The authors have no other conflicts of interest to declare.

Ethical Statement: The authors are accountable for all aspects of the work in ensuring that questions related to the accuracy or integrity of any part of the work are appropriately investigated and resolved. The study was conducted in accordance with the Declaration of Helsinki (as revised in 2013). The study protocol was reviewed and approved by the Ethics Committee of Qingdao Medical College Affiliated to Yantai Yuhuangding Hospital (No. 2014-111), which waived the requirement for informed consent due to the retrospective nature of the study. All the patients provided written informed consent before treatment.

Open Access Statement: This is an Open Access article distributed in accordance with the Creative Commons Attribution-NonCommercial-NoDerivs 4.0 International License (CC BY-NC-ND 4.0), which permits the noncommercial replication and distribution of the article with the strict proviso that no changes or edits are made and the original work is properly cited (including links to both the formal publication through the relevant DOI and the license). See: https://creativecommons.org/licenses/by-nc-nd/4.0/.

\section{References}

1. Bruce DM, Heys SD, Eremin O. Lymphangitis carcinomatosa: a literature review. J R Coll Surg Edinb 1996;41:7-13.

2. Stein MG, Mayo J, Muller N, et al. Pulmonary lymphangitic spread of carcinoma: appearance on CT scans. Radiology 1987;162:371-5.

3. Munk PL, Muller NL, Miller RR, et al. Pulmonary lymphangitic carcinomatosis: CT and pathologic findings. Radiology 1988;166:705-9.

4. Jreige M, Dunet V, Letovanec I, et al. Pulmonary Lymphangitic Carcinomatosis: Diagnostic Performance of High-Resolution CT and 18F-FDG PET/CT in Correlation with Clinical Pathologic Outcome. J Nucl Med 2020;61:26-32.

5. Okazaki A, Shibata K, Matsuda Y, et al. Acute 
Respiratory Failure Caused by Pulmonary Lymphangitic Carcinomatosis in a Patient With Lung Adenocarcinoma at Initial Diagnosis. Arch Bronconeumol 2020;56:534-5.

6. Krasna MJ, White CS, Aisner SC, et al. The role of thoracoscopy in the diagnosis of interstitial lung disease. Ann Thorac Surg 1995;59:348-51.

7. Utz JP, Ryu JH, Douglas WW, et al. High short-term mortality following lung biopsy for usual interstitial pneumonia. Eur Respir J 2001;17:175-9.

8. Wiener RS, Schwartz LM, Woloshin S, et al. Populationbased risk for complications after transthoracic needle lung biopsy of a pulmonary nodule: an analysis of discharge records. Ann Intern Med 2011;155:137-44.

9. Ramaswamy A, Homer R, Killam J, et al. Comparison of Transbronchial and Cryobiopsies in Evaluation of Diffuse Parenchymal Lung Disease. J Bronchology Interv Pulmonol 2016;23:14-21.

10. Torky M, Elshimy WS, Abdelwahab M, et al. Endobronchial ultrasound guided transbronchialcryobiopsy in peripheral lung lesions; efficacy, safety, and comparison with transbronchial forceps biopsy. Clin Respir J 2020. [Epub ahead of print]. doi: 10.1111/crj.13301.

11. Schuhmann M, Bostanci K, Bugalho A, et al. Endobronchial ultrasound-guided cryobiopsies in peripheral pulmonary lesions: a feasibility study. Eur Respir J 2014;43:233-9.

12. Pajares V, Núez-Delgado $M$, Bonet G, et al. Transbronchial biopsy results according to diffuse interstitial lung disease classification. Cryobiopsy versus forceps: MULTICRIO study. PLoS One 2020;15:e0239114.

13. Ramaswamy A, Homer R, Killam J, et al. Comparison of Transbronchial and Cryobiopsies in Evaluation of Diffuse Parenchymal Lung Disease. J Bronchology Interv Pulmonol 2016;23:14-21.

14. Shah UC, Shah AC, Shah CM, editors. Cryo Transbronchial Lung Biopsy Vs Forceps Biopsy Diagnostic Yield and Safety in Interstitial Lung Diseases and Lung Malignancies. Dallas, TX: American Thoracic Society 2019 International Conference, May 17-22, 2019.

15. Hetzel J, Eberhardt R, Herth FJ, et al. Cryobiopsy increases the diagnostic yield of endobronchial biopsy: a multicentre trial. Eur Respir J 2012;39:685-90.

16. Shah U. Efficacy and safety of Cryo transbronchial lung biopsy vs forceps lung biopsy in interstitial lung diseases and lung cancer. Chest 2019;156:A1710.

17. Koslow M, Edell ES, Midthun DE, et al. Bronchoscopic Cryobiopsy and Forceps Biopsy for the Diagnostic
Evaluation of Diffuse Parenchymal Lung Disease in Clinical Practice. Mayo Clin Proc Innov Qual Outcomes 2020;4:565-74.

18. Ganganah O, Guo SL, Chiniah M, et al. Efficacy and safety of cryobiopsy versus forceps biopsy for interstitial lung diseases and lung tumours: A systematic review and meta-analysis. Respirology 2016;21:834-41.

19. Zhan P, Zhu QQ, Miu YY, et al. Comparison between endobronchial ultrasound-guided transbronchial biopsy and CT-guided transthoracic lung biopsy for the diagnosis of peripheral lung cancer: a systematic review and metaanalysis. Transl Lung Cancer Res 2017;6:23-34.

20. Ishida T, Asano F, Yamazaki K, et al. Virtual bronchoscopic navigation combined with endobronchial ultrasound to diagnose small peripheral pulmonary lesions: a randomised trial. Thorax 2011;66:1072-7.

21. Barisione E, Salio M, Romagnoli M, et al. Competence in transbronchial cryobiopsy. Panminerva Med 2019;61:290-7.

22. Hetzel J, Maldonado F, Ravaglia C, et al. Transbronchial Cryobiopsies for the Diagnosis of Diffuse Parenchymal Lung Diseases: Expert Statement from the Cryobiopsy Working Group on Safety and Utility and a Call for Standardization of the Procedure. Respiration 2018;95:188-200.

23. Herth, Felix JF. Bronchoscopy and bleeding risk. Eur Respir Rev 2017;26:170052.

24. Casoni GL, Tomassetti S, Cavazza A, et al. Transbronchial lung cryobiopsy in the diagnosis of fibrotic interstitial lung diseases. PLoS One 2014;9:e86716.

25. Hagmeyer L, Theegarten D, Wohlschlager J, et al. The role of transbronchial cryobiopsy and surgical lung biopsy in the diagnostic algorithm of interstitial lung disease. Clin Respir J 2016;10:589-95.

26. Pourabdollah M, Shamaei M, Karimi S, et al. Transbronchial lung biopsy: the pathologist's point of view. Clin Respir J 2016;10:211-6.

27. Babiak A, Hetzel J, Krishna G, et al. Transbronchial cryobiopsy: a new tool for lung biopsies. Respiration 2009;78:203-8.

28. Ussavarungsi K, Kern RM, Roden AC, et al. Transbronchial Cryobiopsy in Diffuse Parenchymal Lung Disease: Retrospective Analysis of 74 Cases. Chest 2017;151:400-8.

29. Asahina H, Yamazaki K, Onodera Y, et al. Transbronchial biopsy using endobronchial ultrasonography with a guide sheath and virtual bronchoscopic navigation. Chest 2005;128:1761-5. 
30. Asano F, Matsuno Y, Shinagawa N, et al. A virtual bronchoscopic navigation system for pulmonary peripheral lesions. Chest 2006;130:559-66.

31. Iftikhar IH, Alghothani L, Sardi A, et al. Transbronchial Lung Cryobiopsy and Video-Assisted Thoracoscopic Lung Biopsy in the Diagnosis of Diffuse Parenchymal Lung

Cite this article as: Pang L, Jiang T, Liu X, Li Z, Zou S, $\mathrm{Yu}$ P. The value of virtual bronchoscopic navigation and radial endobronchial ultrasound-guided transbronchial lung cryobiopsies for pulmonary lymphangitic carcinomatosis. J Thorac Dis 2020;12(12):7666-7674. doi: 10.21037/jtd-2020abpd-002
Disease: A Meta-analysis of Diagnostic Test Accuracy. Ann Am Thorac Soc 2017;14:1197-211.

32. Bernasconi M, Koegelenberg CFN, Koutsokera A, et al. Iatrogenic bleeding during flexible bronchoscopy: risk factors, prophylactic measures and management. ERJ Open Res 2017;3:00084-2016. 